\title{
ANTIGENIC AND GENOMIC VARIATIONS AMONG CYTOPATHIC AND NON-CYTOPATHIC STRAINS OF BOVINE ENTERIC CORONAVIRUS
}

\author{
S. Dea, L. Michaud, and R. Rekik \\ Centre de Recherche en Virologie \\ Institut Armand Frappier \\ Université du Québec \\ Laval, Québec \\ Canada, H7N $4 Z 3$
}

\section{INTRODUCTION}

Bovine coronavirus (BCV) is a member of the Coronaviridae family known primarly as one of the major causative agent of neonatal calf diarrhea (NCD), a disease with substantial economic impact in the dairy and beef cattle industries ${ }^{1}$. The virus has been reported to be also responsible for acute hemorrhagic enteritis (Winter dysentery) ${ }^{2}$ or mild chronic diarrhea in adult cattle, and for upper respiratory tract illness in growing calves ${ }^{3}$. Although there exist BCV strains associated to different pathological entities in the bovine species, there is still some controversy as to the existence of distinct BCV serotypes. In view of the probable occurrence of pathogenic together with non-pathogenic strains, studies of the determinants that distinguish clinical from subclinical infections are important to establish an adequate control on cattle coronaviral infections. The purpose of the present study was to define the biological, serological and genomic characteristics of $\mathrm{BCV}$ isolates associated with outbreaks of NCD and Winter dysentery in dairy herds in Québec.

\section{METHODOLOGY}

The cell culture-adapted Mebus strain of BCV (BCQ-Meb) ${ }^{1}$ was obtained from the American Type Culture Collection (ATCC-VR874). The reference BCV strain was initially isolated in bovine fetal kidney cells from diarrhea fluid of a calf. Twenty other BCV isolates were recovered from clinical cases of epidemic diarrhea in newborn calves affecting dairy herds located in 4 different geographic areas in Québec ${ }^{4,5}$. Five additional isolates were recovered during winters of 1992 and 1993 from faecal samples collected from adult 
diarrheic cows from herds which were experiencing typical outbreaks of Winter dysentery (WD). No commercial BCV vaccine had been applied in these herds during the year preceeding emergence of clinical cases. The viruses were propagated in the human rectal tumor (HRT-18) cells in the presence of $10 \mathrm{U} / \mathrm{ml}$ of bovine pancreatic trypsin ${ }^{6}$. The extracellular virions were purified by differential and isopycnic ultracentrifugation on continuous 20 to $55 \%(\mathrm{~V} / \mathrm{V})$ sucrose gradients ${ }^{6}$. A rabbit hyperimmune serum was produced against purified tissue culture-adapted BCV-Meb strain. Specificity and cross-reactivity of this antiserum towards Québec BCV isolates were investigated by seroneutralization (SN) and hemagglutination-inhibition $(\mathrm{HI})$ tests $^{7}$. The reactivity of monoclonal antibodies (Mabs) directed against epitopes of the four major antigenic domains of the BCV spike (S) glycoprotein was tested by SN and indirect ELISA ${ }^{4}$.

RT-PCR amplification of the hemagglutinin-esterase (HE) and S genes of the various $\mathrm{BCV}$ isolates were performed as previously described ${ }^{5,8}$. PCR products with A overhangs were ligated into TA cloning vector (pCR II vector; Invitrogen Co.), providing single 3'T overhangs at the insertion site. The ligation mixture was used to transform competent $E$. coli cells. Sequencing of cDNA clones was performed on both strands by the dideoxynucleotide chain-termination method by using T7 DNA polymerase ${ }^{5}$. Sequence analyses were performed using the MacVector 3.5 (International Biotechnologies) and GeneWorks 2.2 (IntelliGenetics Inc) programs.

\section{RESULTS AND DISCUSSION}

Upon their second and third passages in HRT-18 cells, most of clinical BCV isolates induced cytopathic changes within 48 to $72 \mathrm{~h}$ p.i. The various isolates could be differentiated into non-cytopathic (NCP) and cytopathic (CP) strains, the latters being classified into highly fusogenic and non-fusogenic strains. Nevertheless, the yield of viral production was similar for the different isolates (ranged between $10^{55}$ to $10^{700} \mathrm{TCID}_{50} / \mathrm{mL}$ ), as revealed by immuno-peroxydase and calculation of infectivity titers. The five WD isolates behaved as highly fusogenic strains.

All BCV isolates tested were indistinguishable by $\mathrm{SN}$ and Western immunoblotting tests, using rabbit hyperimmune serum to the reference BCV-Meb strain. Interestingly, three $\mathrm{CP}$ isolates could be differentiated by their reactivity to a set of neutralizing Mabs (BCB1, BCF4, BCB5 and BCA3) directed against the $\mathrm{S}$ glycoprotein ${ }^{4}$. Based on competitive ELISA, three antigenic subgroups could be recognized among the BCV isolates studied ${ }^{4}$. These subgroups were identified by Mabs directed to neutralizing epitopes of antigenic domains $\mathrm{A}, \mathrm{B}$ and $\mathrm{C}$ of the $\mathrm{S}$ glycoprotein. Antigenic domain D appeared to be highly conserved among Québec BCV isolates, as well as, non-neutralizing epitopes assigned to antigenic domain $\mathrm{A}$ and $\mathrm{B}$. On the other hand, BCV isolates associated to Winter dysentery could be differentiated by their hemagglutinating activity at $37^{\circ} \mathrm{C}$ and $4^{\circ} \mathrm{C}$, and absence of reactivity to the reference antiserum in the HAI tests.

Oligonucleotide primers, flanking genomic regions coding for the $\mathrm{HE}$ glycoprotein and the S1B immunodominant portion (nt 1185 to 2333) of the S glycoprotein, permitted amplification by PCR of all viruses tested ${ }^{5}$. Sequences of S1B gene fragment of Québec $\mathrm{BCV}$ isolates demonstrated a high degree of similarity. Frameshift, deletion, or insertion, and non sense mutations were not observed; the only variations among these sequences consisted of $40 \mathrm{nt}$ substitutions, which represented $4 \%$ of the sequence ${ }^{5}$. The differing nucleotides were not distributed randomly over the entire sequence but rather were clustered in a highly polymorphic region (nt 1368 to 1776). This was also reflected in the deduced amino acid sequences; indeed, over the S1B sequence most of mutations were silent, whereas in the polymorphic region, most of the nt substitutions resulted in mutations of aa residues. 
The fact, that these aa changes occurred in almost identical location suggests these differences may be significant. Comparative sequence analyses of the highly polymorphic region of the S1 subunit showed that highly cytopathic Québec BCV isolates are genetically divergent from the well characterized L9, F15 and LY-138 strains ${ }^{5}$. Four sporadic aa changes were located in antigenic domain II (aa residues 517 to 720) of their S1 subunits.

Sequence of the predicted proteolytic cleavage site (KRRSRR) was conserved within Québec BCV isolates studied. However, three isolates could be differentiated by their nt sequences proximal to the $\mathrm{S}$ proteolytic cleavage site. Phylogenetic analyses classify recent Québec clinical isolates in a distinct sublineage than other well characterized reference strains $^{5}$.

In conclusion, recent Québec BCV isolates could be differentiated from reference strains by serological and genomic studies, but, no correlation was found between nt and aa substitutions, the rate of viral replication and type of CPE induced in HRT-18 cells. A closer genomic relatedness was demonstrated between the virulent BCV-F15 strain and four highly cytopathogenic Québec isolates. At least 12 aa substitutions have been identified between the virulent and avirulent groups in the highly polymorphic region of the S1 peptide, suggesting that aa changes in this particular region of the $\mathrm{S}$ glycoprotein may be related to $\mathrm{BCV}$ virulence. Nevertheless, we cannot exclude that other regions of the BCV genome may be also involved.

\section{REFERENCES}

1 Mebus C A, Stair E L, Rhodes M B, Twiehaus, M J Neonatal calf diarrhoea propagation, attenuation, and characteristics of a coronavirus-like agent Am J Vet Res 1973,34:145-150

2 Benfield D A, Saif L Cell culture propagation of a coronavirus isolated from cows with winter dysentery J Clin Microbiol 1990,28.1454-1457

3. Reynolds D J , Debney T G, Hall G A , Thomas L H, Parsons K R Studies on the relationship between coronaviruses from the intestınal and respiratory tracts of calves Arch Virol 1985,85.71-83

4 Michaud L, Dea S Characterization of monoclonal antibodies to bovine ente-ric coronavirus and antigenic variations among Quebec isolates Arch Virol 1993, 131 455-465

5. Rekık M.R, Dea S Comparatıve sequence analysis of a polymorphic region of spike protein gene of bovine coronavirus isolates Arch Virol 1994135 319-331

6 Dea S, Garzon S, Tijssen P Isolation and trypsin-enhanced propagation of turkey enteric (Bluecomb) coronaviruses in a contınuous HRT-18 cell line Am J Vet Res 1989,50 1310-1318

7 Dea S, Tijssen P Antıgenic and polypeptide structure of turkey enteric coronaviruses as defined by monoclonal antibodies J Gen Virol 1989,70 1725-1741

8 Zhang X, Kousoulas K G, Storz J Comparıson of the nucleotıde and deduced amino acid sequences of the S genes specified by virulent and avirulent strains of bovine coronaviruses Virology 1991,183 397 404 